\title{
DISCUSSION
}

\section{Upper-bound solutions for face stability of circular tunnels in undrained clays}

\author{
FEI ZHANG*, YUFENG GAO*, YONGXIN WU*, NING ZHANG*, BOONCHAI UKRITCHON†, \\ KONGKIT YINGCHALOENKITKHAJORN $\ddagger$ and SURAPARB KEAWSAWASVONG $\dagger$
}

\section{Contribution by B. Ukritchon, K. Yingchaloenkitkhajorn and S. Keawsawasvong}

This discussion is based on the paper by Zhang et al. (2018). The authors presented three-dimensional (3D) upper-bound (UB) solutions for undrained face stability of circular tunnels based on the kinematic approach of limit analysis, where a continuous velocity field with a toric envelope was adopted. The authors validated the derived UB solutions against that of Klar \& Klein (2014) for a constant strength profile, and presented the stability charts of the stability number $N$ affected by the dimensionless parameters $C / D$, and $\rho D / s_{\mathrm{u} 0}$. Finally, an approximate formula for $N_{\mathrm{c} 0}$ and $N_{\mathrm{c} \rho}$ was proposed in equation (28) in the original paper for a calculation of the critical pressure.

Recently, the discussers published a paper in Computers and Geotechnics entitled 'Three-dimensional undrained tunnel face stability in clay with a linearly increasing shear strength with depth' (Ukritchon et al., 2017). Unlike the authors' approach, 3D finite-element analysis (FEA) was employed by the discussers to study only the collapse of the tunnel face. In that paper, a non-linear regression was also employed to statically derive $N_{\mathrm{c} 0}$ and $N_{\mathrm{c} \rho}$ from the finite-element (FE) solutions with a coefficient of determination $\left(R^{2}\right)$ of $99 \cdot 99 \%$, and an algebraic formula of these was proposed. However, a comparison between the work of the authors and Ukritchon et al. (2017) was not given in the paper under discussion. Thus, it seems appropriate to write this discussion that addresses the comparison between them regarding the derived $N_{\mathrm{c} 0}$ and $N_{\mathrm{c} \rho}$ and the proposed approximate formula of these.

Figure 10 shows a comparison of $N_{\mathrm{c} 0}$ for tunnels in clays with constant strength profile $(\rho=0)$ between the paper under discussion, Ukritchon et al. (2017) and Mollon (2012). Note that the latter is based on 3D UB calculations using a continuous velocity field with a toric failure mechanism, and was simulated by Ukritchon et al. (2017) using the Matlab toolbox, tunnel face stability software (TFSS) (Mollon, 2012), which represents the most up-to-date of previous studies by Mollon et al. (2009, 2010, 2011, 2013). Since the UB analysis with the same failure mechanism of toric shape was employed by both the authors and Mollon (2012) and the UB solution of the former is higher than that of the latter by about $17-24 \%$, the $N_{\mathrm{c} 0}$ solution in the paper under

\footnotetext{
* Key Laboratory of Ministry of Education for Geomechanics and Embankment Engineering, Hohai University, Nanjing, P. R. China. $\dagger$ Geotechnical Research Unit, Department of Civil Engineering, Faculty of Engineering, Chulalongkorn University, Bangkok, Thailand.

+ Department of Civil Engineering, College of Industrial Technology and Management, Rajamangala University of Technology Srivijaya, Songkhla, Thailand.
}

discussion is moderately inaccurate. For $C / D \leq 1 \cdot 5$, there is excellent agreement in $N_{\mathrm{c} 0}$ between Mollon (2012) and Ukritchon et al. (2017). For $C / D \geq 2 \cdot 0$, these two solutions diverge and the latter predicts a lower $N_{\mathrm{c} 0}$ ratio. For $C / D=0 \cdot 5-5, N_{\mathrm{c} 0}$ of Ukritchon et al. (2017) is smaller than that of the paper under discussion by approximately $20-36 \%$. Fig. 11 shows a comparison of $N_{\mathrm{c} \rho}$ for tunnels in clays with linearly increasing strength profile $(\rho=0)$. Note that there is no solution of Mollon (2012) in this case since TFSS is restricted to an analysis of the tunnel face in homogeneous clays. In general, both the results of Ukritchon et al. (2017) and the paper under discussion predict that $N_{\mathrm{c} \rho}$ increases non-linearly with $C / D$, where the latter is always plotted above the former by approximately $32-45 \%$. To justify which $N_{\text {c } \rho}$ is more accurate, a lower-bound solution of the problem is needed. However, it can be inferred from the result in Fig. 10 that the $N_{\mathrm{c} \rho}$ of the paper under discussion is likely to be inaccurate, as in the case of $N_{\mathrm{c} 0}$.

The second issue of the discussion is related to the selected mathematical form of the approximate formula for $N_{\mathrm{c} 0}$ and $N_{\mathrm{c} \rho}$ used in the original paper, where a third-order polynomial function was employed, as shown in equation (28). Unlike that form, Ukritchon et al. (2017) employed a power function to regress the FEA results with a coefficient of determination $\left(R^{2}\right)$ of $99 \cdot 99 \%$, as

$$
\begin{aligned}
& N_{\mathrm{c} 0}=a_{1}\left(\frac{C}{D}\right)^{a_{2}} \\
& N_{\mathrm{c} \rho}=a_{3}\left(\frac{C}{D}\right)^{a_{4}}
\end{aligned}
$$

where $a_{1}=7 \cdot 8835, a_{2}=0 \cdot 3365, a_{3}=7 \cdot 6072$ and $a_{4}=1 \cdot 2489$.

It should be noted that the regression range with $C / D=0.5-5$ and $N_{\gamma}=C / D+0 \cdot 5$ were adopted in the regression analyses by both Ukritchon et al. (2017) and the authors. Figs 12 and 13 show comparisons of $N_{\mathrm{c} 0}$ and $N_{\mathrm{c} \rho}$ between the two papers, respectively, particularly when $C / D$ is outside the range of regression (i.e. $C / D \geq 5$ ). It can be observed from Fig. 12 that $N_{\mathrm{c} 0}$ in the original paper changes its curvature for $C / D \geq 5$. In contrast, the power function employed by Ukritchon et al. (2017) gives a gradual increase in $N_{\mathrm{c} 0}$ for all $C / D$ ratios that are both inside and outside the range of the regression. For example, at $C / D=10$, the original paper predicts $N_{\mathrm{c} 0}$ that is 2.7 times higher than that of Ukritchon et al. (2017). This result implies that the stability solution calculated from the original paper will be too high, thereby likely predicting an unsafe limiting surcharge $\left(\sigma_{\mathrm{s}}\right)$ for $C l D \geq 5$. As shown in Fig. 13, it is apparent that $N_{\mathrm{c} \rho}$ in the original paper is a concave function with a global maximum at $C / D$ of about 13 , and hence produces an unrealistic prediction for $C / D \geq 13$. On the contrary, such an unusual result is not observed for $N_{\mathrm{c} \rho}$ of 


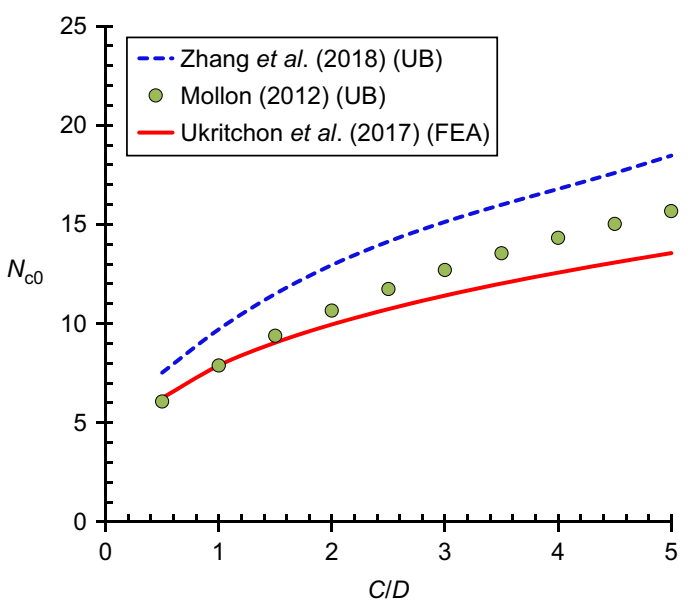

Fig. 10. Comparison of $N_{\mathrm{c} 0}$ for $C I D=0 \cdot 5-5$

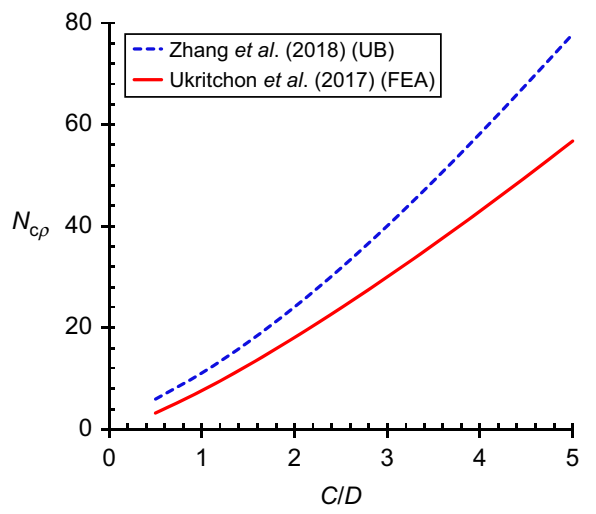

Fig. 11. Comparison of $N_{\mathrm{c} \rho}$ for $C I D=0 \cdot 5-5$

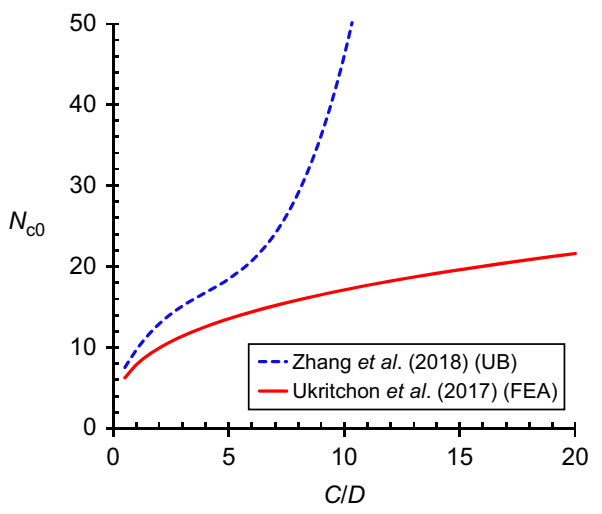

Fig. 12. Comparison of $N_{\mathrm{c} 0}$ for $C I D=0 \cdot 5-20$

Ukritchon et al. (2017). Consequently, it can be concluded that the cubic functions selected by the authors are unsuitable for accurately and realistically describing both $N_{\mathrm{c} 0}$ and $N_{\mathrm{c} \rho}$ for $C / D \geq 5$ that is outside the range of the regression. Thus, practitioners should be aware of this limitation. In contrast, the power function for $N_{\mathrm{c} 0}$ and $N_{\mathrm{c} \rho}$ proposed by Ukritchon et al. (2017) in equation (32) above is much simpler and requires fewer constant terms than the cubic function in the original paper, and yields a conservative and reliable prediction on these stability factors as described earlier.

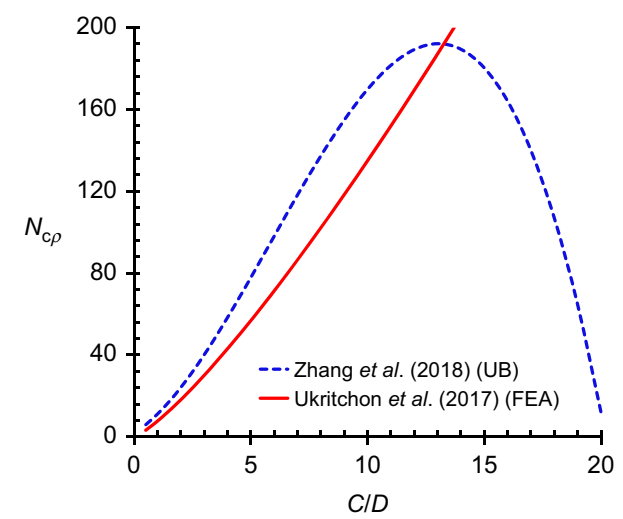

Fig. 13. Comparison of $N_{\mathrm{c} \rho}$ for $C I D=0 \cdot 5-20$

\section{Authors' reply}

The authors appreciate the comments of the discussers. All the issues raised by the discussers have been addressed as follows.

First of all, this note does not make a comparison of the derived UB solutions with the numerical FE results given by the discussers (Ukritchon et al., 2017). This note (received 10 September 2016; accepted 14 March 2017) was accepted a little earlier than the discussers' paper (received 18 December 2016; accepted 19 March 2017). Unfortunately, the authors could not give the comparisons.

Second, the discussers made the comparisons of the critical support pressure on the face of the circular tunnel in undrained clay. For constant undrained strength with depth, the presented comparison of the UB solutions between Mollon et al. (2013) and this study demonstrates that the solutions of this study are less critical than those derived by Mollon et al. (2013). The reason for this has been explained by Klar \& Klein (2014) as that the non-rigorous definition of the strain rate tensor in Mollon et al. (2013) may yield a lower value of the stability number $N_{\mathrm{c} 0}$. Therefore, this study adopted the closed-form analytical expression of the curvilinear strain rate tensor presented by Klar \& Klein (2014) to calculate the least UB solutions of the support pressure. The comparison between Klar \& Klein (2014) and this study has been given to show the good agreement of the calculated results, as shown in Table 1. The obtained UB solutions of this study are definitely accurate.

Next, the discussers compared their numerical FE results with the UB solutions of this study and demonstrate the criticality of support pressure obtained from the FE method. The FE results could be affected by the boundary conditions, the mesh refinement and the selection of the limit state and cannot be regarded as a rigorous solution for a problem in the limit state. The kinematic approach of limit analysis (LA) is based on the UB theorem of plasticity. The obtained UB solution is rigorous but its criticality depends on the constructed kinematically admissible velocity field. For the face stability of a tunnel in undrained clay, the continuous velocity field proposed by Mollon et al. (2013) may not yield the least UB solution in LA and can be further improved.

Finally, the discussers extended the application of the presented approximate formula based on the third-order polynomial fitting function to the case for larger ratio of the cover depth to the tunnel diameter $(5 \leq C / D \leq 20)$. However, the fitting values of the coefficients $P_{0}, P_{1}, P_{2}$ and $P_{3}$ from the UB solutions for $C / D$ ranging from 0 to 5 are used for the comparisons by the discussers. In fact, the suggested values of these coefficients given in Table 3 are only limited to the case for $C / D$ ranging from 0 to 5 . As the discussers stated, such a limitation should be given for clarity in practical use. For the larger ratios $C / D$, the authors obtained the UB solutions of $N_{\mathrm{c} 0}$ 


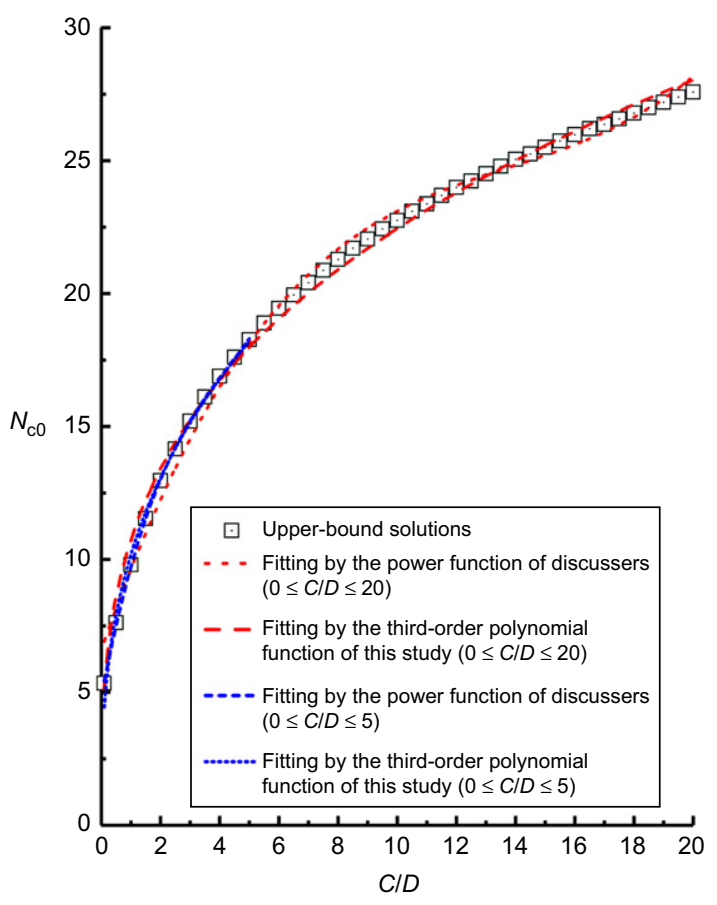

(a)

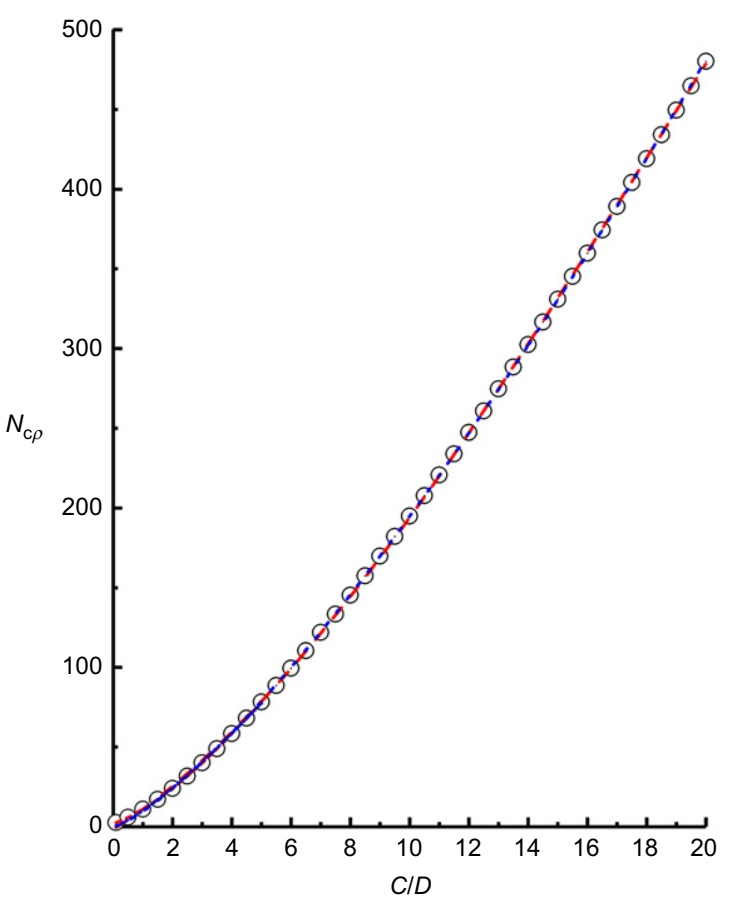

(b)

Fig. 14. Comparisons of the fitting UB results between the power function of the discussers and the third-order polynomial function of the study under discussion

Table 4. Results determined by different regression functions

\begin{tabular}{|c|c|c|c|c|c|c|}
\hline \multirow[t]{2}{*}{ Regression functions } & \multirow[t]{2}{*}{$C / D$} & \multirow{2}{*}{$\begin{array}{c}\text { Fitting } \\
\text { coefficients }\end{array}$} & \multicolumn{2}{|r|}{$N_{\mathrm{c} 0}$} & \multicolumn{2}{|r|}{$N_{\mathrm{c} \rho}$} \\
\hline & & & Value & Statistical coefficient $R^{2}$ & Value & Statistical coefficient $R^{2}$ \\
\hline $\begin{array}{l}\text { Third-order polynomial function } \\
\text { of this study }\end{array}$ & $\begin{array}{l}{[0,5]} \\
{[0,20]}\end{array}$ & $\begin{array}{l}P_{0} \\
P_{1} \\
P_{2} \\
P_{3} \\
P_{0} \\
P_{1} \\
P_{2} \\
P_{3}\end{array}$ & $\begin{array}{r}4 \cdot 8659 \\
5 \cdot 8187 \\
-1 \cdot 0703 \\
0 \cdot 0861 \\
6 \cdot 6051 \\
3 \cdot 2155 \\
-0 \cdot 2063 \\
0 \cdot 0050\end{array}$ & $\begin{array}{l}0.9996 \\
0.9937\end{array}$ & $\begin{array}{r}1 \cdot 8388 \\
7 \cdot 0609 \\
2 \cdot 3339 \\
-0 \cdot 1363 \\
-1 \cdot 5494 \\
11.6699 \\
0.9589 \\
-0.0171\end{array}$ & $0 \cdot 9999$ \\
\hline Power function of discussers & $\begin{array}{l}{[0,5]} \\
{[0,20]}\end{array}$ & $\begin{array}{l}a_{1} \\
a_{2} \\
a_{1} \\
a_{2}\end{array}$ & $\begin{array}{r}10 \cdot 1847 \\
0 \cdot 3605 \\
10 \cdot 7306 \\
0 \cdot 3206\end{array}$ & $\begin{array}{l}0.9934 \\
0.9959\end{array}$ & $\begin{array}{r}10 \cdot 1622 \\
1 \cdot 2627 \\
9 \cdot 6396 \\
1 \cdot 3053\end{array}$ & $\begin{array}{l}0.9986 \\
0.9999\end{array}$ \\
\hline
\end{tabular}

and $N_{\mathrm{c} \rho}$, as shown in Fig. 14. Their trends remain consistent with the FE results given by the discussers. Using the same function to regress the extended results can determine the values of those coefficients, as shown in Table 4. It can be seen that the statistical coefficient $R^{2}$ is still 0.9999 for $N_{\mathrm{c} \rho}$, but slightly reduces for $N_{\mathrm{c} 0}$. Generally, the presented third-order polynomial regression function is in good agreement with the obtained UB solutions, as shown in Fig. 14. In addition, the authors also employ the power function suggested by the discussers to regress the UB results and obtain the values of the coefficients $a_{1}$ and $a_{2}\left(a_{3}\right.$ and $a_{4}$ for $\left.N_{\mathrm{c} \rho}\right)$ in equation (32), as shown in Table 4 . When the ratio is less than 5 , the value of the statistical coefficient $R^{2}$ obtained by the power function is less than that derived from the third-order polynomial function. Therefore, the third-order polynomial fitting function is more suitable for the UB solutions of this study. In practice, the cover depth is usually less than five times the tunnel diameter.

In summary, the note aims to determine the UB solutions of the critical support pressure on the circular face of the tunnel in undrained clay in which undrained strength increases linearly with depth. The continuous velocity field proposed by Mollon et al. (2013) is adopted here to calculate the UB solutions in LA. However, this postulated velocity field can be further improved for better estimation on the UB solutions. The approximate formula, based on the third-order polynomial regression function fitting to the UB solutions, provides an alternative tool for quick estimation of the critical supporting pressure. It should be noted that the suggested values of the fitting coefficients in Table 3 are limited to the case for $C / D$ ranging from 0 to 5 .

Again, the authors of this note thank the discussers for their interest in this study.

\section{REFERENCES}

Klar, A. \& Klein, B. (2014). Energy-based volume loss prediction for tunnel face advancement in clays. Géotechnique 64, No. 10, 776-786, https://doi.org/10.1680/geot.14.P.024.

Mollon, G. (2012). Tunnel face stability software (TFSS): ver. 1.0. Lyon, France: INSA Lyon, University of Lyon. See http:// guilhem.mollon.free.fr/Accueil_Eng.html (accessed 15/09/2015).

Mollon, G., Dias, D. \& Soubra, A. (2009). Probabilistic analysis and design of circular tunnels against face stability. Int. J. Geomech. $A S C E$ 9, No. 6, 237-249. 
Mollon, G., Dias, D. \& Soubra, A. (2010). Face stability analysis of circular tunnels driven by a pressurized shield. J. Geotech. Geoenviron. Engng ASCE 136, No. 1, 215-229.

Mollon, G., Dias, D. \& Soubra, A. (2011). Rotational failure mechanisms for the face stability analysis of tunnels driven by a pressurized shield. Int. J. Numer. Analyt. Methods Geomech. 35, No. 12, 1363-1388.

Mollon, G., Dias, D. \& Soubra, A. (2013). Continuous velocity fields for collapse and blowout of a pressurized tunnel face in purely cohesive soil. Int. J. Numer. Analyt. Methods Geomech. 37, No. 13, 2061-2083.

Ukritchon, B., Yingchaloenkitkhajorn, K. \& Keawsawasvong, S. (2017). Three-dimensional undrained tunnel face stability in clay with a linearly increasing shear strength with depth. Comput. Geotech. 88, 146-151.

Zhang, F., Gao, Y. F., Wu, Y. X. \& Zhang, N. (2018). Upper-bound solutions for face stability of circular tunnels in undrained clays. Geotechnique 68, No. 1, 76-85, https://doi.org/10. 1680/jgeot.16.T.028. 